Postgrad. Med. J. (1965), 41, 2

\title{
THE DIAGNOSIS OF APPENDICITIS
}

\author{
P. H. Sмiтh, M.B. (Leeds), F.R.C.S.
}

Resident Surgical Officer, St. James' Hospital, Leeds

DESPITE the fact that appendicitis is the commonest general surgical emergency, every surgeon is aware that its diagnosis is frequently difficult and this study has been made to review the significance of the symptoms and signs which have been stated to be of value in its differential diagnosis.

The classical syndrome of "central abdominal pain, anorexia, nausei, vomiting, pain in the right iliac fossa, fever and leucocytosis" presents no problems. However, the syndrome is incomplete in many cases and in some the only relevant feature is pain or tenderness in the right iliac fossa.

\section{Method}

A proforma was designed to record the patient's symptoms and signs on admission, the operative findings and post-operative sourse and included the following information:-

\section{History :-}

1. Sex, age and presenting symptoms.

2. Pain:-duration, site, type, radiation, aggravating and relieving factors.

3. Anorexia, nausea and vomiting.

4. Bowels, micturition and menstrual history.

5. History of cold, sore throat, headaches, previous similar attacks and previous illnesses.

\section{Findings:-}

1. Temperature and pulse: condition of throat, tongue and breath.

2. Abdominal examination for tenderness, rigidity, palpable mass, bowel sounds, psoas, obturator and Sherren tests, Rovsing's sign and the results of a rectal examination.

3. Investigations when done-white cell count (WBC), midstream urine (MSU) and erythrocyte sedimentation rate (ESR).

Operative Findings and Treatment

Post-operative Course

A prospective survey was carried out including patients in the series if it was felt that

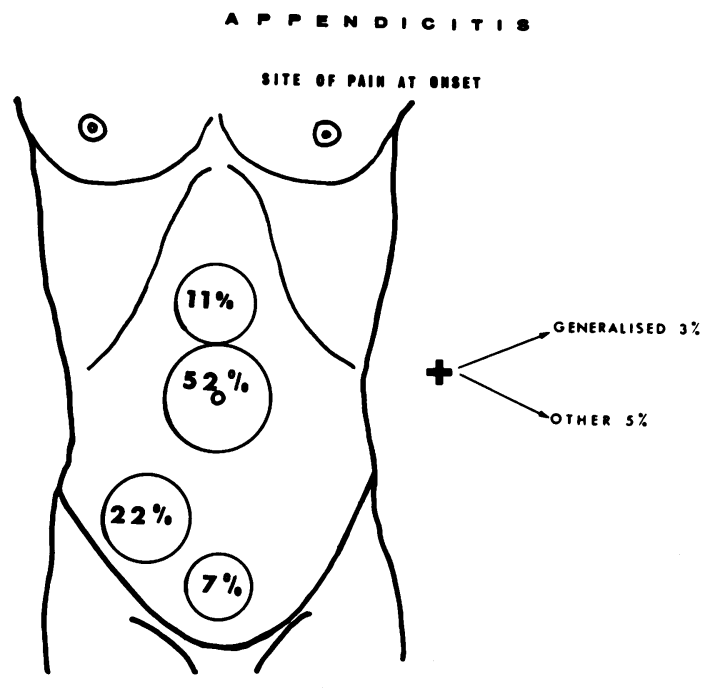

FIG. 1.-Site of pain at onset

appendicitis could not be excluded, i.e. that operation was required, and the series was continued until 100 patients with a proven diagnosis of appendicitis were available for discussion. The initial diagnosis was incorrect in approximately $20 \%$ of cases and thus the series numbers 124 cases in all. There were no deaths.

\section{Results}

Of the 100 cases of appendicitis, 59 were male and 41 female. Their ages ranged from 2 to 80 years, threequarters $(77 \%)$ being as expected under 30 years of age. 94 patients suffered pain as the presenting symptom, whilst of the remaining 6 , two first complained of anorexia, two of nausea and vomiting, and one of headache.

Pain. This was described as colicky by 60 patients, as constant by 28 , and in the remaining 12 it was either "indescribable" or absent.

Its site at onset was as shown in Figure 1, and it subsequently radiated to the right iliac fossa in 58 patients, elsewhere in 10 and not at all in 32 . 


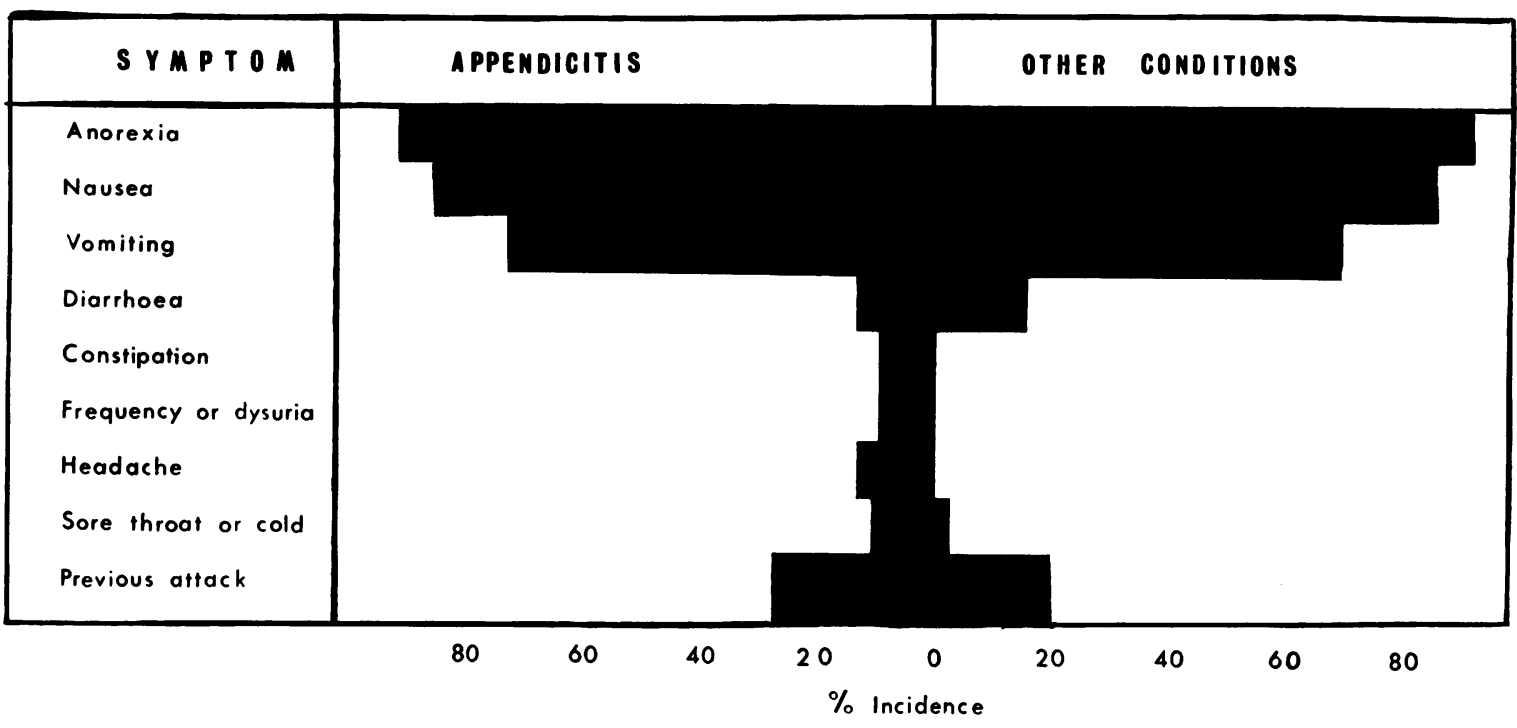

TABLE 1.-Symptoms of patients in the series

The other complaints, together with their incidence, are listed in Table 1 , and it will be noted that the findings in those with and without appendicitis are very similar. No patient showed significant menstrual abnormality and it was noted that 24 patients had some previous but irrelevant illness, e.g. coronary thrombosis, jaundice, hernia repair, etc.

The clinical findings are recorded in Table 2 and it will again be noted that the incidence of the respective findings in those with and without appendicitis is very similar. One might also mention that only $60 \%$ of those with appendicitis had a temperature of $99^{\circ} \mathrm{F}$ or more and, even more important, in $28 \%$ it was $98^{\circ} \mathrm{F}$ or less.

\section{Investigations}

In the earlier cases and in some of the more difficult later cases, a white cell count, MSU and ESR were performed.

1. White Cell Count-was undertaken in 30 cases, being over $10,000 / \mathrm{cu}$. mm. in 20 cases, all of whom had appendicitis. Of the remaining 10 patients - all of whom had a normal white count-six had appendicitis.

2. $E S R$ - was carried out in 24 cases and proved of no value at all.
3. $M S U$-was taken in 19 cases. Of these 4 were abnormal and showed:-

(i) 10 red cells/high power field (HPF).

(ii) Frequent pus cells.

(iii) 10-15 white cells and 4 red cells/HPF.

(iv) 20-30 white cells/HPF.

All these patients had appendicitis, the appendix being pelvic and gangrenous in the latter two instances.

\section{Discussion}

A mere glance at the tables of results shows that none of the classical features-apart from tenderness in the right iliac fossa-was invariably present and that the incidence of the majority was no higher in those with appendicitis than it was in those suffering from some other condition. Of the investigations, a white cell count may be helpful if it is abnormal but an MSU seems to be positively dangerous, since it may falsely suggest disease of the urinary tract. The ESR was of no value.

How then is a diagnosis of appendicitis to be made-if it can be made-and what principles should be used in the management of patients with suspected appendicitis?

\section{Diagnosis}

Since the pain of appendicitis can start in, 


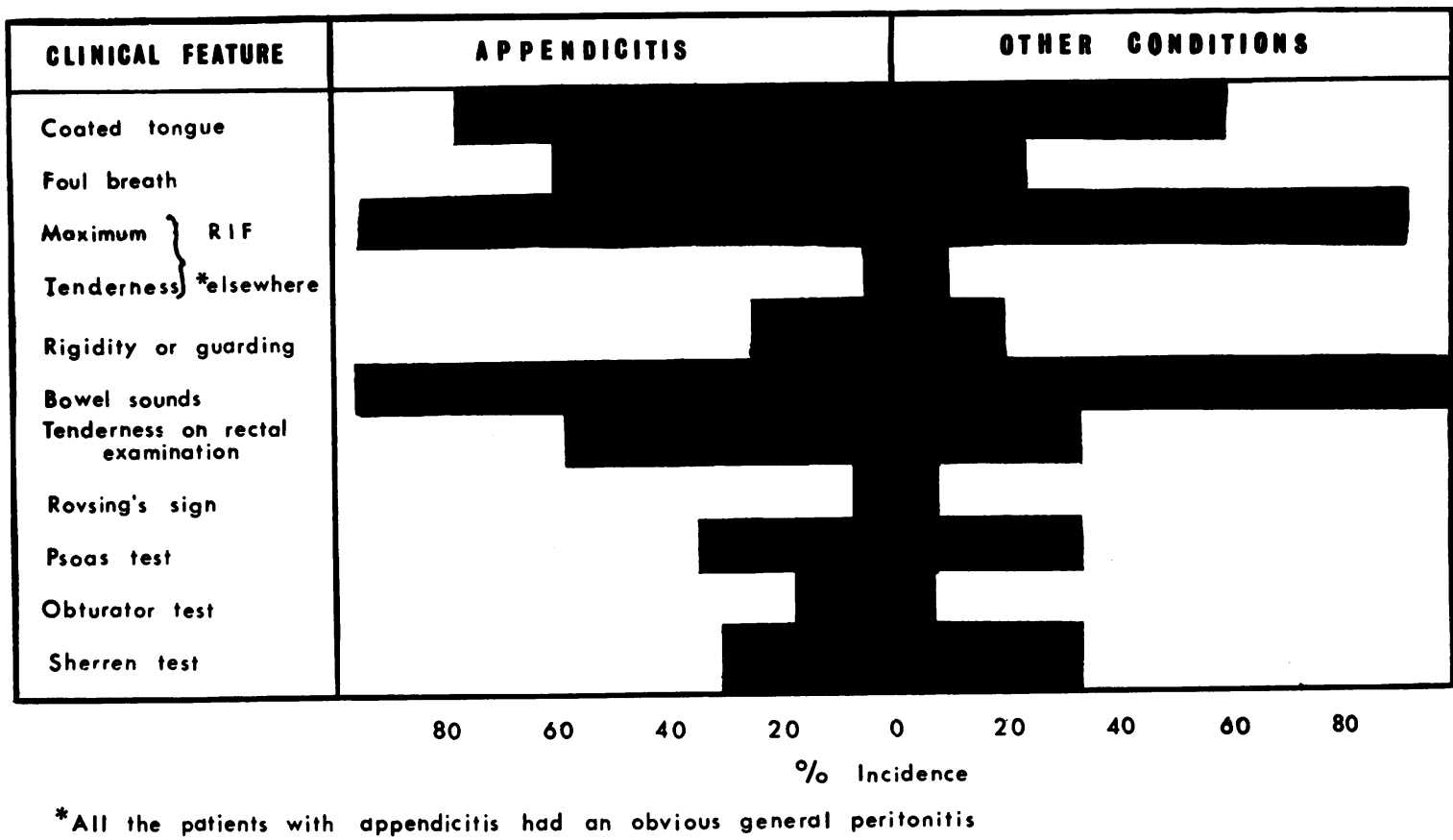

TABLE 2.-Clinical findings in patients in the series.

and radiate to, virtually any part of the abdomen, it is impossible to make or exclude the diagnosis with absolute certainty from a detailed history of the pain. Also, as the incidence of the majority of symptoms and signs is equal in those with and without appendicitis, there appears to be no cardinal feature of the condition.

In these circumstances one can only consider those features which occur in the vast majority of cases of appendicitis, e.g., over $70 \%$, and say that in their absence appendicitis is much less likely. Using these criteria the features of appendicitis emerge, as expected, as abdominal pain moving to or arising in the right iliac fossa, associated with anorexia, nausea, vomiting, tenderness in the right iliac fossa and a leucocytosis.

Unfortunately, however, this picture is by no means diagnostic of appendicitis ( $v$. infra) and in any event is found in only two thirds of the cases of appendicitis, the remaining third being anomalous, presenting as Boyes (1949) states "a widely variable syndrome, a confused and disorderly syndrome, in which almost any symptoms and signs may appear in almost any sequence". In short, it is impossible to diagnose appendicitis with certainty. The truth of this statement will be seen by the following examples:

1. Of 3 patients with a 24-30 hour history $\overrightarrow{\overrightarrow{0}}$ of colicky central abdominal pain radiating to the right iliac fossa and associated with anorexia, nausea, vomiting, fever and tenderness in the right iliac fossa and on rectal examination 흑 -one had appendicitis, one mesenteric lymphadenitis and the third a leaking chocolate cyst of the right ovary.

2. On the other hand, three other patients who had respectively no pain in the right iliac $ᄋ$ fossa and pain in the epigastrium, all had appendicitis, the only common feature being tenderness in the right iliac fossa.

\section{Management}

Even though the features of appendicitis are so variable as to be, in many cases, of little value in diagnosis there still remain two large groups of cases:-

1. Those with a "typical" history of appendicitis-some of whom $(12.5 \%$ in this series) will not in fact have appendicitis.

2. Those with an atypical history of appen-

.


dicitis-many of whom ( $68 \%$ in this series) will in fact have appendicitis.

In the first group operation must obviously be advised in every case since it is far less dangerous to remove a few normal appendices than to leave even one inflamed and possibly gangrenous appendix in situ.

In the second group the diagnosis will be correct in only 2 cases in every 3 and it is in this group that the decision whether to operate or not may well be difficult. Here the deciding factor must be the presence or absence of tenderness in the right iliac fossa. It was present in all cases of appendicitis in this series and, in its presence, one cannot exclude the possibility of appendicitis.

I would suggest, therefore, that in all patients with abdominal pain who have neither a palpable mass nor obvious gynaecological or urinary disease, the presence of tenderness in the right iliac fossa demands immediate operation and that only by so doing will one prevent the serious or even fatal consequences of misdiagnosis of the more difficult cases of appendicitis.

\section{Summary}

A prospective review of 100 case of appen- dicitis was carried out. It is pointed out yet again, as often in the past, that it is impossible to diagnose appendicitis with certainty since the clinical features are variable and may be mimicked by other conditions. A white count may be of help if raised but an MSU may be positively misleading and should be carried out only to confirm a clinical diagnosis of urinary tract disease. The special tests such as Rovsing's sign, psoas and obturator tests and Sherren's test are not helpful and medical students would probably be better employed learning other things.

The only constant finding in this series of patients with abdominal pain was tenderness in the right iliac fossa and it is suggested that this finding in the absence of a palpable mass or obvious urinary or gynaecological disease merits operation whether or not the history is consistent with appendicitis.

I should like to thank Mr. D. B. Feather, Mr. A. J. C. Latchmore, the late Mr. M. C. Oldfield, Mr. J. H. Shoesmith, Mr. H. S. Shucksmith and $\mathrm{Mr}$. G. Wilson for permission to publish these cases and Mr. Howard and Mr. Lang for the illustrations.

\section{REFERENCE}

Boyce, F. F. (1949): In "Acute Appendicitis and its Complications", page 154, New York: Oxford 